\title{
Calidad de los quistes de Artemia persimilis (Piccinelli \& Prosdocimi) (Crustacea: Branchiopoda) de diferentes zonas de Argentina, como alimento en acuicultura
}

\author{
Quality of Artemia persimilis (Piccinelli \& Prosdocimi) (Crustacea: Branchiopoda) \\ cysts from different zones of Argentina as food in aquaculture \\ Natalia E. Sato ${ }^{1,4}$, Juan C. Mallo ${ }^{2,4}$ y Jorge L. Fenucci ${ }^{3,4}$ \\ ${ }^{1}$ Departamento de Ciencias Marinas \\ ${ }^{2}$ CIC (Comisión de Investigaciones Científicas, provincia de Buenos Aires). Departamento de Ciencias Marinas \\ ${ }^{3}$ CONICET. Departamento de Ciencias Marinas \\ ${ }^{4}$ Universidad Nacional de Mar del Plata. Funes 3250, 7600 Mar del Plata, Argentina \\ nesato@mdp.edu.ar
}

\begin{abstract}
Resumen.- Se evaluó la calidad de los quistes de Artemia persimilis (Piccinelli \& Prosdocimi 1968) procedentes de las salinas de las provincias de Buenos Aires, La Pampa y Córdoba. Para ello, se determinaron las tallas de huevos, número de quistes por gramo, tallas de nauplios, porcentajes y tiempos de eclosión, tiempos de sincronía $\left(\mathrm{T}_{\mathrm{s}}\right)$, luego del tratamiento con $\mathrm{H}_{2} \mathrm{O}_{2}$ y el análisis de la calidad nutricional de los quistes en lo referente a proteínas, lípidos totales, ácidos grasos, cenizas y humedad.

Los resultados fueron satisfactorios, con porcentajes de eclosión que variaron, en general, entre un $60 \%$ y $80 \%$ y $\mathrm{T}_{\mathrm{s}}$ que no superaron las 7,5 h. El diámetro promedio con el desvío estándar de los quistes hidratados, tuvo valores entre $184,7 \pm 26,29 \mu \mathrm{m}$ (Salina El Chancho) y 248,6 $\pm 17,29 \mu \mathrm{m}$ (Salina Colorada Grande). El número de quistes por gramo varió entre 102.600 (Salinas Grandes de Anzoátegui) y 276.000 (Salinas Grandes de Hidalgo). La talla promedio con el desvío estándar de los nauplios varió entre 350,6 \pm 36,01 $\mu \mathrm{m}$ (Salina El Chancho) y 512,1 \pm 63,44 $\mu \mathrm{m}$ (Salina Colorada Grande). El tratamiento de los quistes con $\mathrm{H}_{2} \mathrm{O}_{2}$, redujo los $\mathrm{T}_{\mathrm{s}}$. Con respecto a la calidad nutricional, se obtuvieron valores comparables a los que se comercializan actualmente. Se puede afirmar que los quistes de Artemia persimilis podrían ser utilizados como alimento en acuicultura.
\end{abstract}

Palabras clave: Crustáceos, nutrición, alimento vivo

\begin{abstract}
The cysts quality of Artemia persimilis (Piccinelli \& Prosdocimi 1968) from the salines of the provinces of Buenos Aires, La Pampa and Cordoba, was evaluated. Eggs size, number of cysts per gram, nauplius size, hatching percentages, hatching rate, synchrony time $\left(T_{s}\right)$ after the treatment with $\mathrm{H}_{2} \mathrm{O}_{2}$ and analysis of the nutritional quality the cysts regarding protein, total lipids, fatty acids, ash and moisture were determined.

The results were satisfactory, with hatching percentages varying, in general, from $60 \%$ to $80 \%$ and with Ts that didn't overcome $7.5 \mathrm{~h}$. The average diameter with the standard deviation of the hydrated cysts, were between $184.7 \pm 26.29$ $\mu \mathrm{m}$ (Salina El Chancho) and 248.6 $17.29 \mu \mathrm{m}$ (Salina Colorada Grande). The number of cysts per gram varied from 102,600 (Salinas Grandes de Anzoátegui) to 276,000 (Salinas Grandes de Hidalgo). The average size, with the standard deviation of the nauplius, varied from $350.6 \pm 36.01 \mu \mathrm{m}$ (Salina El Chancho) to 512.1 $463.44 \mu \mathrm{m}$ (Salina Colorada Grande). The cyst treatment with $\mathrm{H}_{2} \mathrm{O}_{2}$ reduced the time of synchrony. With regard to the nutritional quality, the values obtained can be compared to the ones presently traded. It can be affirmed that the cysts of Artemia persimilis could be used as food in aquaculture.
\end{abstract}

Key words: Crustacean, brine shrimp, nutrition, live food

\section{Introducción}

Con el desarrollo que ha adquirido la acuicultura en todo el mundo, el uso de Artemia como alimento vivo para las larvas en cultivo, llegó a ser generalizado (Lavens \& Sorgeloos 2000).
El género Artemia tiene la capacidad de producir quistes, a partir de los cuales, se obtienen fácilmente por eclosión, estadios naupliares. Es por ello que Artemia constituye el alimento vivo más utilizado para las formas larvarias de peces y crustáceos (Navarro et al. 1999). Según De los Ríos \& Gajardo (2002) se cosechan alrededor de 3.000 y 1.000 toneladas de quistes y 
biomasa, respectivamente, siendo el principal productor de quistes, Estados Unidos de América (Gran Lago Salado).

La calidad de los quistes depende de un gran número de factores, tales como la calidad nutricional intrínseca, las características de la diapausa, la talla de los quistes y nauplios, entre otras, que influencian en el valor comercial de los mismos (Bossier et al. 2004).

Según Lavens \& Sorgeloos (1996) otros de los criterios que también definen la calidad de los quistes de Artemia como alimento en acuicultura son: los porcentajes y tiempos de eclosión, los tiempos de sincronía y el número de quistes por gramo.

El $90 \%$ de los quistes que se consumen a nivel mundial, provienen del Gran Lago Salado (Utah, Estados Unidos) (Castro et al. 2000), pero desafortunadamente la producción de este sitio ha mermado de tal forma por los cambios climáticos sufridos en el medio, que se hace necesario localizar y evaluar nuevas poblaciones de Artemia, que contribuyan al abastecimiento de los mercados nacionales e internacionales.

En la República Argentina existen abundantes biotopos hipersalinos propios para la presencia de poblaciones de Artemia (Angelelli et al. 1976, Angelelli et al. 1980, Schalamuk et al. 1983, Schalamuk et al. 1999). En Argentina se encuentra la especie Artemia persimilis (Piccinelli \& Prosdocimi 1968). Algunos autores como Cohen et al. (1999) y Lavens \& Sorgeloos (2000), indican que merece considerable atención la evaluación de este recurso natural con destino a la acuicultura.

El objetivo de este trabajo fue analizar la calidad de los quistes de Artemia persimilis como fuente de alimento en camaronicultura y piscicultura. Para ello, se determinaron las tallas de quistes y nauplios, el porcentaje y tiempo de eclosión, el tiempo de sincronía luego del tratamiento con solución de peróxido de hidrógeno $\left(\mathrm{H}_{2} \mathrm{O}_{2}\right)$ para lograr la desactivación de la diapausa y la calidad nutricional de los quistes en lo referente a proteínas, lípidos totales, ácidos grasos, cenizas y humedad.

\section{Materiales y métodos}

El material analizado provino de las siguientes salinas de la Argentina:

\section{Provincia de Buenos Aires}

Salinas Chicas (3846’s, 6259’W), situada aproximadamente a $110 \mathrm{~km}$ al norte de la localidad de Pedro Luro y Salina del Inglés (4043’S, 62²9’W), situada aproximadamente a $40 \mathrm{~km}$ al sudeste de Cardenal Cagliero (Fig. 1) ( Schalamuk et al. 1999).

\section{Provincia de La Pampa}

Salinas Grandes de Anzoátegui (3900’S, 6347’W), situada a $5 \mathrm{~km}$ al sudeste de la localidad de Anzoátegui y a $24 \mathrm{~km}$ al norte del río Colorado; salina El Chancho (38³7’S, 6545’ W), situada al norte de la localidad de Anzoátegui; Salitral Negro (3840’S, 6408’W) situada a $45 \mathrm{~km}$ al norte de la localidad de La Adela; Salina

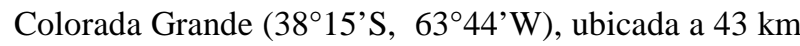
al sudoeste de General San Martín; Salina Colorada Chica (38²3'S, 6336'W), situada a $35 \mathrm{~km}$ al sur de Jacinto Arauz (departamento Caleu Caleu) y Salina Grandes de Hidalgo (37²1'S, 63³5’W), ubicada al sudeste de la localidad de Macachín (departamento Atreuco) (Fig. 1) (Schalamuk et al. 1999).

\section{Provincia de Córdoba}

Salinas Grandes de Córdoba, localizada en el ángulo noroccidental de la provincia de Córdoba entre los

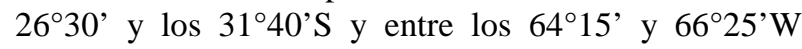
(Fig. 2) (Dargám 1995).

La recolección de los quistes se realizó en las orillas de las salinas donde éstos se acumulan. Una vez recogidos, se ubicaron en baldes con salmuera y de esta manera fueron transportados hasta el laboratorio para su posterior estudio. Inicialmente cada muestra fue lavada con una solución con 200 g/L de sal y pasada a través de tamices de 400 y $180 \mu \mathrm{m}$ de malla. Previamente al secado, los quistes se ubicaron en un recipiente con salmuera (200 g/L de sal) para separar los quistes que flotaron en la superficie de la salmuera, de la fracción pesada compuesta principalmente por arena, que precipitó en el fondo. Posteriormente, los quistes flotantes se recogieron y se pasaron a un recipiente con agua destilada. Los quistes viables se depositaron en el fondo y luego se sometieron a secado en estufa a $35^{\circ} \mathrm{C}$ hasta peso constante (Amat 1980). La medición de la talla de los quistes, se realizó dejando hidratar previamente los mismos, en un recipiente con agua destilada a temperatura ambiente, durante 2 h, bajo luz constante utilizando una lámpara de 60 watts. Luego se procedió a la medición de los diámetros, 


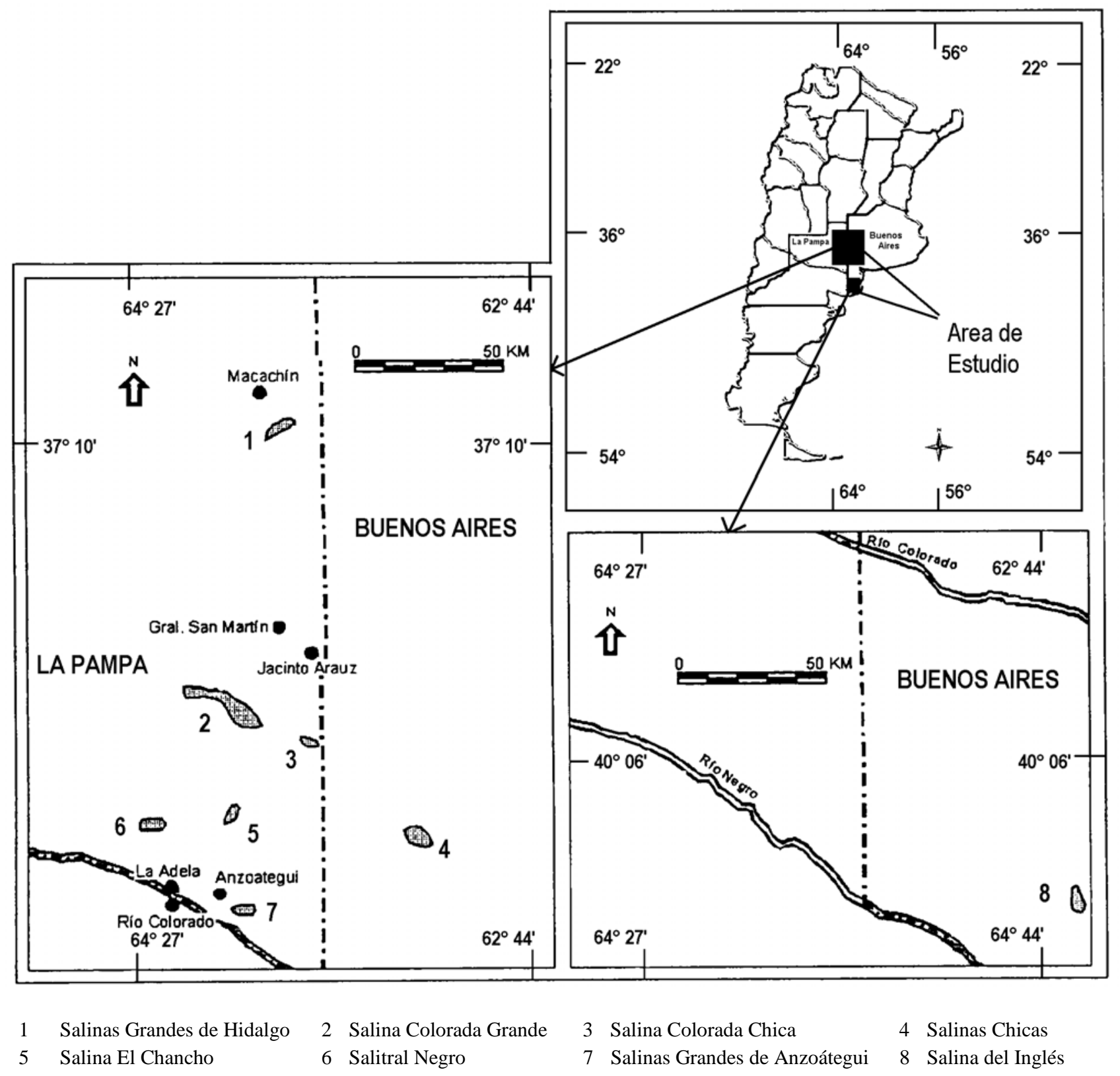

Figura 1

Mapa con la localización de las salinas estudiadas de las provincias de La Pampa y Buenos Aires, Argentina. Modificado de Schalamuk et al. (1999)

Map with location of the salines studied of La Pampa and Buenos Aires Argentina. Modified from Schalamuk et al. (1999) 


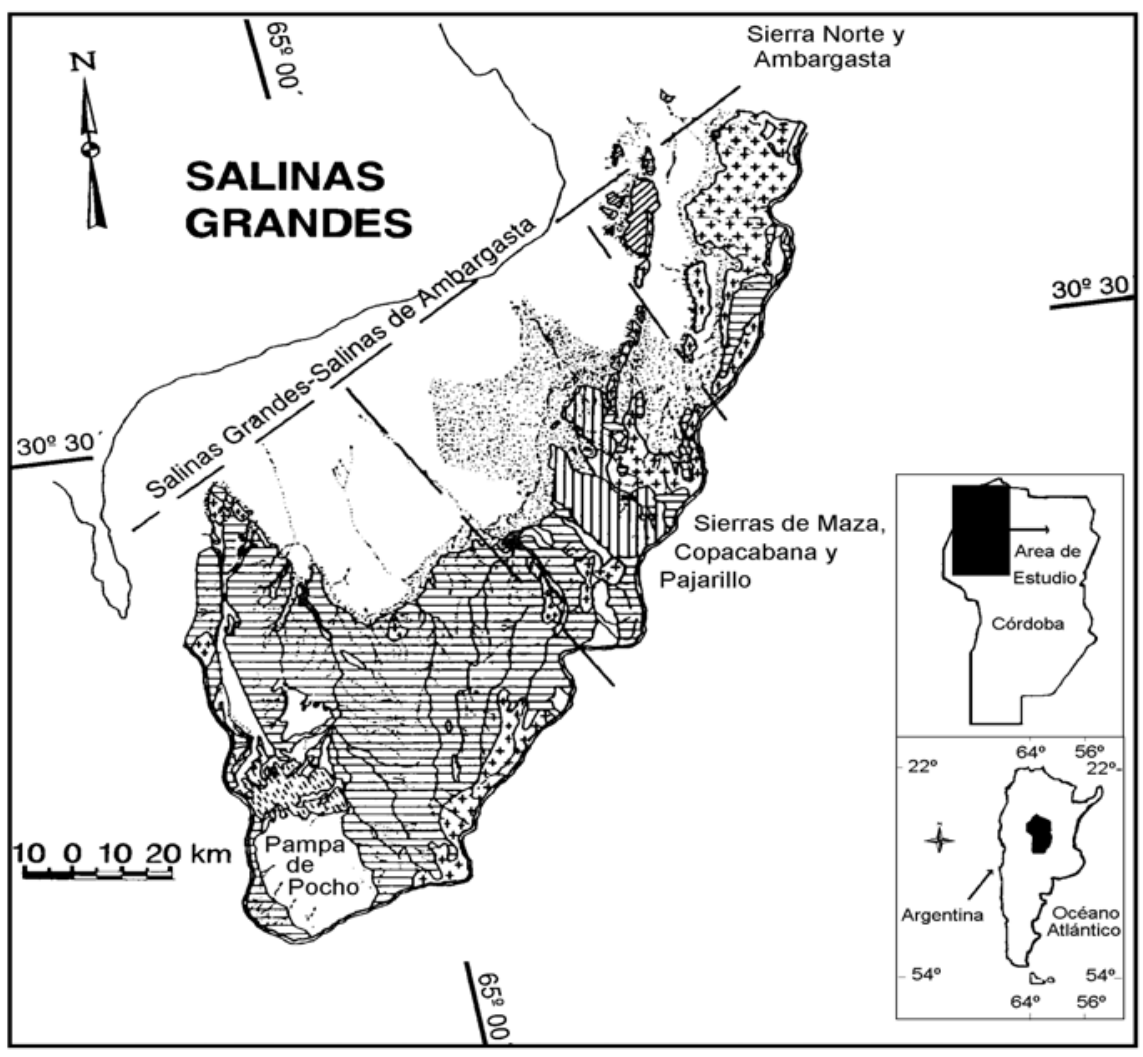

Figura 2

\section{Mapa con la localización de las Salinas Grandes de Córdoba, provincia de Córdoba, Argentina. Modificado de Dargám (1995)}

Map with location of the Salinas Grandes de Córdoba, province of Córdoba, Argentina. Modified from Dargám (1995)

utilizando una lupa dotada de un ocular micrométrico. De cada una de las muestras se midieron 30 quistes. Para la determinación del número de quistes por gramo, se pesó, con balanza analítica con una precisión de $\pm 0,001 \mathrm{~g}$, una submuestra de $10 \mathrm{mg}$ de quistes secos, para cada una de las muestras y se realizó el conteo bajo lupa binocular. En la medición de las tallas de nauplios recién eclosionados, se hidrataron 200 quistes de cada una de las muestras en agua destilada durante $2 \mathrm{~h}$ y luego se incubaron en recipientes plásticos transparentes en las condiciones estándares. Luego a $\mathrm{T}_{90}$ (horas desde el inicio de la incubación hasta el $90 \%$ del total de nauplios), se procedió a la medición de 30 nauplios de cada muestra, utilizando una lupa dotada de ocular micrométrico. La tinción de las muestras se realizó con solución de Lugol al 5\% y se midieron desde la cabeza hasta el final del abdomen excluyendo las setas.

Para determinar los porcentajes y tiempos de eclosión de los quistes, se procedió según las técnicas descriptas por Lavens \& Sorgeloos (1996). De cada una de las muestras se realizaron tres réplicas. Para determinar los tiempos de sincronía luego de la desactivación de la diapausa, se dejaron hidratar quistes de cada muestra durante $2 \mathrm{~h}$ en agua dulce a temperatura ambiente y se transfirieron a una solución de peróxido de hidrógeno $\left(\mathrm{H}_{2} \mathrm{O}_{2}\right)$ al $5 \%$ durante $5 \mathrm{~min}$, en recipientes provistos de aireación para homogeneizar el medio. Luego, se enjuagaron con abundante agua destilada para eliminar las trazas del compuesto. Los quistes sometidos al tratamiento, fueron incubados en las condiciones estándares. Los quistes también fueron tratados, para comparar, con una solución de $\mathrm{H}_{2} \mathrm{O}_{2}$ al 0,5\% y $2 \%$.

\section{Análisis estadísticos}

A los datos obtenidos se les calculó la media con su desvío estándar. Los datos fueron sometidos a las siguientes pruebas estadísticas: ANOVA con un 5\% de significancia; las condiciones previas que se requieren para el ANOVA son: normalidad (prueba de bondad de ajuste), que las muestras sean independientes (prueba de independencia) y homocedasticidad (prueba de Bartlett) (Sokal \& Rohlf 1979), prueba de comparaciones múltiples de Tukey $(p<0,05)$ según Zar (1984), empleando el programa Statistica 5.0. 
Para estimar la calidad nutricional de los quistes, se llevó a cabo el análisis de proteínas, lípidos totales, ácidos grasos, cenizas y humedad. En todos los casos, con excepción del análisis de humedad, se trabajó con quistes descapsulados empleando las técnicas descriptas por Lavens \& Sorgeloos (1996). El análisis de proteínas se realizó según Barnes (1959); en el de lípidos totales se procedió según IRAM (1985) y el de los ácidos grasos se realizó según Moreno (1977). Para la cuantificación del contenido de cenizas de los quistes, se empleó la técnica descripta por Moreno \& Aizpun (1969). El contenido de humedad en los quistes se determinó según la metodología empleada por Horwitz (1960). En todos los casos, de cada muestra se realizaron tres réplicas. A los datos obtenidos se les calculó la media con su desvío estándar.

\section{Resultados}

\section{Diámetro de quistes hidratados, números de quistes por gramo y talla de nauplios de Artemia persimilis}

Los datos del diámetro de los quistes hidratados, el número de quistes por gramo y la talla de nauplios recién eclosionados de Artemia persimilis, provenientes de las diferentes salinas analizadas, se muestran en la Tabla 1. La prueba de comparaciones múltiples de Tukey mostró que los diámetros de los quistes de las muestras procedentes de Colorada Grande (248,6 $\pm 17,29 \mu \mathrm{m})$, fueron significativamente mayores $(p<0,05)$, cuando se compararon con las muestras de las otras salinas, excepto las de Salinas Chicas $(242,4 \pm 18,82 \mu \mathrm{m})(p=0,981988)$ y Salinas Grandes de
Córdoba $(232,5 \pm 21,64 \mu \mathrm{m}) \quad(p=0,146146)$ que no presentaron diferencias significativas. Las tallas de los quistes de Salinas Chicas $(242,4 \pm 18,82 \mu \mathrm{m})$ fueron también significativamente mayores $(p<0,05)$ cuando se compararon con las muestras de las otras salinas, excepto las de Colorada Grande (248,6 \pm 17,29 $\mu \mathrm{m})$ ( $p=0,981988)$ y Salinas Grandes de Córdoba $(232,5 \pm 21,64 \mu \mathrm{m}) \quad(p=0,768571) \quad$ donde no se observaron diferencias significativas. Con respecto a los diámetros de los quistes de Salinas Grandes de Córdoba (232,5 $\pm 21,64 \mu \mathrm{m})$, éstos fueron significativamente mayores $(p<0,05)$ cuando se compararon con las muestras procedentes de Salitral Negro (214,4 \pm 24,04 $\mu \mathrm{m})$, Salinas Grandes de Hidalgo (212,2 \pm 24,90 $\mu \mathrm{m})$, Salina del Inglés $(212,0 \pm 32,72 \mu \mathrm{m})$, Colorada Chica $(210,0 \pm 34,80 \mu \mathrm{m})$ y El Chancho $(184,7 \pm 26,70 \mu \mathrm{m})$. Con relación a los diámetros de los quistes procedentes de Salinas Grandes de Anzoátegui (215,9 \pm 26,70 $\mu \mathrm{m})$ se observaron diferencias significativas $(p<0,05)$ entre las muestras procedentes de Colorada Grande (248,6 \pm 17,29 $\mu \mathrm{m})$, Salinas Chicas $(242,4 \pm 18,82 \mu \mathrm{m})$ y El Chancho $(184,7 \pm 26,70 \mu \mathrm{m})$. Las tallas de los quistes procedentes de Salitral Negro (214,4 $\pm 24,04$ $\mu \mathrm{m})$, Salinas Grandes de Hidalgo (212,2 $\pm 24,90 \mu \mathrm{m})$, Salina del Inglés $(212,0 \pm 32,72 \mu \mathrm{m})$ y Colorada Chica $(210,0 \pm 34,80 \mu \mathrm{m})$ mostraron valores significativamente menores $(p<0,05)$ en comparación con las muestras de Colorada Grande (248,6 \pm 17,29 $\mu \mathrm{m})$, Salinas Chicas $(242,4 \pm 18,82 \mu \mathrm{m})$ y Salinas Grandes de Córdoba (232,5 $\pm 21,64 \mu \mathrm{m})$. Hay que destacar, que las tallas de los quistes procedentes de la salina El Chancho fueron significativamente menores $(p<0,05)$ en comparación con el resto de las salinas analizadas.

\section{Tabla 1}

Talla de quistes hidratados, número de quistes por gramo y talla de nauplios de Artemia persimilis provenientes de las diferentes salinas analizadas. Los datos corresponden a la media con su desvío estándar de 3 réplicas

Size of hydrated cysts, number of cysts per gram and size of nauplii of Artemia persimilis from the different salines. Data correspond to average and standard deviation of 3 replicates

\begin{tabular}{|c|c|c|c|}
\hline \multirow[t]{2}{*}{ Salinas } & Diámetro del quiste hidratado $(\mu \mathrm{m})$ & Talla de nauplios $(\mu \mathrm{m})$ & \multirow[t]{2}{*}{ Número de quistes por gramo } \\
\hline & $\bar{X} \quad s$ & $\bar{X} \quad s$ & \\
\hline Colorada Grande & $248,6 \pm 17,29$ & $512,1 \pm 63,44$ & 221.000 \\
\hline Salinas Chicas & $242,4 \pm 18,82$ & $469,5 \pm 26,51$ & 240.550 \\
\hline Salinas Grandes de Córdoba & $232,5 \pm 21,64$ & $466,8 \pm 24,60$ & 241.050 \\
\hline Salinas Grandes de Anzoátegui & $215,9 \pm 26,70$ & $426,7 \pm 54,03$ & 102.600 \\
\hline Salitral Negro & $214,4 \pm 24,04$ & $366,0 \pm 23,30$ & 160.550 \\
\hline Salinas Grandes de Hidalgo & $212,2 \pm 24,90$ & $429,2 \pm 70,05$ & 276.000 \\
\hline Salina del Inglés & $212,0 \pm 32,72$ & $402,5 \pm 41,65$ & 186.000 \\
\hline Colorada Chica & $210,0 \pm 34,80$ & $372,3 \pm 26,68$ & 271.100 \\
\hline El Chancho & $184,7 \pm 26,70$ & $350,6 \pm 36,01$ & 137.833 \\
\hline
\end{tabular}


Los resultados de la prueba de comparaciones múltiples de Tukey, mostraron que las tallas de los nauplios procedentes de Colorada Grande $(512,1 \pm 63,44 \mu \mathrm{m})$ fueron significativamente mayores $(p<0,05)$ cuando se compararon con las muestras de las otras salinas estudiadas. La talla de los nauplios procedentes de Salinas Chicas $(469,5 \pm 26,51 \mu \mathrm{m})$ y Salinas Grandes de Córdoba (466,8 $\pm 24,60 \mu \mathrm{m})$, no mostraron diferencias significativas $(p=1,000000)$ entre ellas. Las tallas de los nauplios procedentes de Salinas Grandes de Anzoátegui (426,7 $\pm 54,03 \mu \mathrm{m})$ y Salinas Grandes de Hidalgo $(429,2 \pm 70,05 \mu \mathrm{m})$ fueron significativamente menores $(p<0,05)$ al ser comparados con las tallas de los nauplios procedentes de Colorada Grande $(512,1 \pm 63,44 \mu \mathrm{m})$, Salinas Grandes de Córdoba $(466,8 \pm 24,60 \mu \mathrm{m}) \quad$ y Salinas Chicas $(469,5 \pm 26,51 \mu \mathrm{m})$. En cambio se observó, que las tallas naupliares de Salinas Grandes de Anzoátegui $(426,7 \pm 54,03 \mu \mathrm{m})$ y Salinas Grandes de Hidalgo $(429,2 \pm 70,05 \mu \mathrm{m})$ fueron significativamente mayores $(p<0,05)$ cuando se compararon con las tallas de nauplios procedentes de Salitral Negro (366,0 \pm 23,30 $\mu \mathrm{m})$, Colorada Chica $(372,3 \pm 26,68 \mu \mathrm{m})$ y El Chancho (350,6 $\pm 36,01 \mu \mathrm{m})$. Las tallas naupliares procedentes de Salitral Negro $(366,0 \pm 23,30 \mu \mathrm{m})$, no mostraron diferencias significativas con las tallas naupliares provenientes de Salina del Inglés (402,5 \pm 41,65 $\mu \mathrm{m})$ ( $\mathrm{P}=0,058778)$, Colorada Chica $(372,3 \pm 26,68 \mu \mathrm{m})$ $(\mathrm{P}=0,999996) y \quad$ El Chancho $(350,6 \pm 36,01 \mu \mathrm{m})$ $(\mathrm{P}=0,787980)$. Con respecto a las tallas de los nauplios provenientes de la Salina del Inglés (402,5 \pm 41,65 $\mu \mathrm{m})$, se encontraron que fueron significativamente menores $(p<0,05)$ al ser comparadas con las muestras provenientes de Colorada Grande (512,1 \pm 63,44 $\mu \mathrm{m})$, Salinas Chicas $(469,5 \pm 26,51 \mu \mathrm{m})$ y Salinas Grandes de Córdoba $(466,8 \pm 24,60 \mu \mathrm{m})$. Las tallas naupliares correspondientes a Colorada Chica $(372,3 \pm 26,68 \mu \mathrm{m})$ no mostraron diferencias significativas al ser comparadas con las tallas naupliares procedentes de Salitral Negro $(366,0 \pm 23,30 \mu \mathrm{m}) \quad(\mathrm{P}=0,999996)$ y Salina del Inglés $(402,5 \pm 41,65 \mu \mathrm{m})(\mathrm{P}=0,140089)$. Con respecto al número de quistes por gramo de muestra seca y limpia, varió entre 102.600 (Salinas Grandes de Anzoátegui) y 276.000 (Salinas Grandes de Hidalgo).

\section{Porcentaje de eclosión, tiempo de eclosión y tiempo de sincronía de los quistes de Artemia persimilis}

Se determinaron los porcentajes de eclosión de los quistes de Artemia persimilis de las distintas salinas, como indicativo de la viabilidad de los mismos. Los porcentajes de eclosión de los quistes, variaron entre
$82,0 \pm 2,00 \%$ (Salinas Chicas) y 42,0 $\pm 2,83 \%$ (El chancho). Los valores más bajos y por lo tanto indicativo de una menor calidad, correspondieron a El Chancho (42,0 $\pm 2,83 \%)$ Salinas Grandes de Anzoátegui (44,0 $\pm 6,93 \%)$ y Colorada Grande (45,3 $\pm 8,39 \%)$ (Tabla 2). No se observaron diferencias significativas $(p<0,05)$ en los porcentajes de eclosión de los quistes provenientes de Salinas Chicas $(82,0 \pm 2,00 \%)$ y Salinas del Inglés $(75,7 \pm 4,29 \%)$. Si se observaron diferencias significativas $(p<0,05)$ entre los porcentajes de eclosión de los quistes provenientes de Salina del Inglés (75,7 $\pm 4,29 \%)$ y las siguientes salinas: Salitral Negro (62,3 \pm 7,77\%), Salinas Grandes de córdoba (61,7 $\pm 7,64 \%)$, Colorada Grande (45,3 $\pm 8,39 \%)$, Salinas Grandes de Anzoátegui

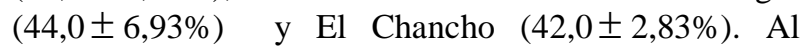
comparar los porcentaje de eclosión de los quistes procedentes de Colorada Chica (70,0 \pm 7,50\%), Salinas Grandes de Hidalgo (70,0 $\pm 2,00 \%)$, Salitral Negro $(62,3 \pm 7,77 \%)$ y Salinas Grandes de Córdoba $(61,7 \pm 7,64 \%)$ no se observaron diferencias, pero si se observaron diferencias significativas $(p<0,05)$ entre estas salinas y los porcentajes de eclosión de los quistes procedentes de las restantes salinas analizadas. Los porcentajes de eclosión de quistes procedentes de Colorada Grande (45,3 \pm 8,39\%), Salinas Grandes de Anzoátegui (44,0 $\pm 6,93 \%)$ y El Chancho $(42,0 \pm 2,83 \%)$, no mostraron diferencias significativas $(p<0,05)$ entre si. Se determinaron los tiempos de eclosión y los tiempos de sincronía $\left(\mathrm{T}_{\mathrm{s}}\right)$ de los quistes de Artemia persimilis de las distintas salinas, obteniéndose los siguientes resultados que se muestran en la Tabla 2. Con relación al tiempo de sincronía, los análisis estadísticos mostraron que los $\mathrm{T}_{\mathrm{s}}$ de los quistes provenientes de Salinas Chicas $(4,7 \pm 0,29 \mathrm{~h})$ fueron significativamente menores $(p<0,05)$ en comparación con los $\mathrm{T}_{\mathrm{s}}$ de los quistes procedentes de Salina del Inglés (7,0 $\pm 1,00 \mathrm{~h})$, Colorada Chica (7,0 $\pm 1,00 \mathrm{~h}) \mathrm{y}$ Salinas Grandes de Córdoba (7,3 \pm 0,58 h). Los $\mathrm{T}_{\mathrm{s}}$ de los quistes procedentes de El Chancho $(5,5 \pm 0,70 \mathrm{~h})$ fueron significativamente menores $(p<0,05)$ al ser comparados con los $\mathrm{T}_{\mathrm{s}}$ de los quistes provenientes de Salinas Grandes de Córdoba (7,3 $\pm 0,58 \mathrm{~h})$. Los $\mathrm{T}_{\mathrm{s}}$ de los quistes de Salinas Grandes de Córdoba (7,3 \pm 0,58 h) fueron significativamente mayores $(p<0,05)$ al ser comparados con los $\mathrm{T}_{\mathrm{s}}$ de los quistes de Salinas Chicas $(4,7 \pm 0,29 \mathrm{~h})$ y El Chancho $(5,5 \pm 0,70 \mathrm{~h})$. Los $\mathrm{T}_{\mathrm{s}}$ de los quistes procedentes de las Salinas Grandes de Hidalgo (5,5 0,87 h), Salitral Negro (6,2 \pm 0,29 h), Colorada Grande (6,2 \pm 0,58 h) y Salinas Grandes de Anzoátegui (6,3 $\pm 0,58 \mathrm{~h})$ no mostraron diferencias entre ellos, ni con el resto de las salinas estudiadas. 
Tabla 2

Tiempo de eclosión (horas), tiempo de sincronía (horas) y porcentaje de eclosión de los quistes de Artemia persimilis de las distintas salinas estudiadas. Los datos corresponden a la media con su desvío estándar de 3 réplicas

Hatching time (hours), time of synchrony (hours) and hatching percentage of the cysts of Artemia persimilis from the different salines studied. The data correspond to average and standard deviation of 3 replicates

\begin{tabular}{cccccc}
\hline Salinas & $\mathrm{T}_{0}(\mathrm{~h})$ & $\mathrm{T}_{10}(\mathrm{~h})$ & $\mathrm{T}_{90}(\mathrm{~h})$ & $\begin{array}{c}\text { Tasa de sincronía } \\
\left(\mathrm{T}_{\mathrm{s}}\right)\end{array}$ & $\begin{array}{c}\text { Porcentaje de } \\
\text { eclosión (\%) }\end{array}$ \\
\hline Salinas Chicas & $16,0 \pm 0,87$ & $18,3 \pm 1,44$ & $23,0 \pm 1,73$ & $4,7 \pm 0,29$ & $82,0 \pm 2,00$ \\
El Chancho & $17,5 \pm 2,12$ & $20,0 \pm 2,83$ & $25,5 \pm 3,53$ & $5,5 \pm 0,70$ & $42,0 \pm 2,83$ \\
Salina Grandes de Hidalgo & $17,2 \pm 0,29$ & $18,8 \pm 1,04$ & $24,3 \pm 1,89$ & $5,5 \pm 0,87$ & $70,0 \pm 2,00$ \\
Salitral Negro & $16,7 \pm 2,31$ & $19,3 \pm 1,15$ & $25,5 \pm 1,32$ & $6,2 \pm 0,29$ & $62,3 \pm 7,77$ \\
Colorada Grande & $17,3 \pm 1,15$ & $19,3 \pm 1,15$ & $25,5 \pm 1,73$ & $6,2 \pm 0,58$ & $45,3 \pm 8,39$ \\
Salinas Grandes de Anzoátegui & $17,5 \pm 0,87$ & $19,3 \pm 1,15$ & $25,7 \pm 0,58$ & $6,3 \pm 0,58$ & $44,0 \pm 6,93$ \\
Salina del Inglés & $16,7 \pm 0,58$ & $18,0 \pm 1,00$ & $25,0 \pm 2,00$ & $7,0 \pm 1,00$ & $75,7 \pm 14,29$ \\
Colorada Chica & $16,3 \pm 0,58$ & $18,3 \pm 1,04$ & $25,3 \pm 2.02$ & $7,0 \pm 1,00$ & $70,0 \pm 7,50$ \\
Salinas Grandes de Córdoba & $16,2 \pm 1,04$ & $18,0 \pm 1,80$ & $25,3 \pm 2,25$ & $7,3 \pm 0,58$ & $61,7 \pm 7,64$ \\
\hline
\end{tabular}

$\mathrm{T}_{0}$ : horas desde el inicio de la incubación hasta el primer nauplio

$\mathrm{T}_{10}$ : horas desde el inicio de la incubación hasta el $10 \%$ del total de nauplios

$\mathrm{T}_{90}$ : horas desde el inicio de la incubación hasta el $90 \%$ del total de nauplios

$\mathrm{T}_{\mathrm{s}}$ : tiempo de sincronía o tasa de eclosión: $\mathrm{T}_{90}-\mathrm{T}_{10}$

\section{Tiempo de sincronía de los quistes de Artemia persimilis luego del tratamiento con peróxido de hidrógeno para lograr la desactivación de la diapausa}

Los resultados de la determinación de los tiempos de sincronía $\left(\mathrm{T}_{\mathrm{s}}\right)$ de los quistes de las diferentes salinas estudiadas mediante el tratamiento con $\mathrm{H}_{2} \mathrm{O}_{2}$ y su posterior incubación, se muestran en las Figs. 3 y 4.

El gráfico correspondiente a Salina del Inglés, muestra un tiempo de sincronía $\left(\mathrm{T}_{\mathrm{s}}\right)$ significativamente más bajo $(p<0,05)(4,2 \pm 0,29 \mathrm{~h})$, y por lo tanto mejor, cuando se aplicó a los quistes, una dosis del $5 \%$ de $\mathrm{H}_{2} \mathrm{O}_{2}$ en comparación al $\mathrm{T}_{\mathrm{s}}$ logrado con las otras dosis suministradas (Fig. 3).

El gráfico correspondiente a Salinas Grandes de Hidalgo, muestra un mejoramiento significativo de la sincronía, cuando se aplicó una dosis del 0,5\% del compuesto, $\left(T_{s}\right.$ de 4,3 $\left.\pm 0,28 \mathrm{~h}\right)$ en comparación al logrado con dosis más altas (Fig. 3).

El gráfico correspondiente a Salinas Chicas, muestra un mejoramiento significativo $(p<0,05)$ del $\mathrm{T}_{\mathrm{s}}$ cuando los quistes fueron tratados con una dosis al $0,5 \%$ de
$\mathrm{H}_{2} \mathrm{O}_{2}$, presentando un $\mathrm{T}_{\mathrm{s}}$ de $4,2 \pm 0,29$ h. No sucedió lo mismo con aplicaciones de dosis más altas de $\mathrm{H}_{2} \mathrm{O}_{2}$ (Fig. 3). Con respecto al resto de las salinas estudiadas, no se observaron diferencias significativas $(p<0,05)$ en los tiempos de sincronía entre los diferentes tratamientos (Figs. 3 y 4 ).

\section{Calidad nutricional de los quistes de Artemia persimilis}

Se realizó el análisis de la composición de las proteínas, lípidos totales, cenizas y humedad. Los resultados se muestran en la Tabla 3. Se obtuvieron valores similares entre las salinas analizadas, en relación al contenido de proteínas, lípidos totales, cenizas y humedad en quistes. En la Tabla 4 se muestran los resultados de la composición porcentual de los ácidos grasos a partir de quistes de Artemia persimilis obtenidos de dos salinas distintas: Salinas Chicas y Salinas Grandes de Córdoba. Se observaron niveles elevados de ácido linolénico (18: 3 (n-3)) en los quistes procedentes de ambas salinas. En Salinas Chicas fue del 23,1\% y en Salinas Grandes de Córdoba fue del 19,62\%. 
Salina del Inglés

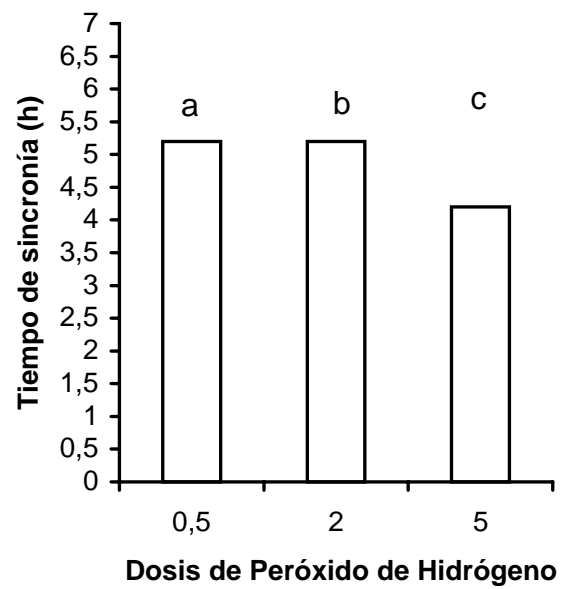

(\%)

Salitral Negro

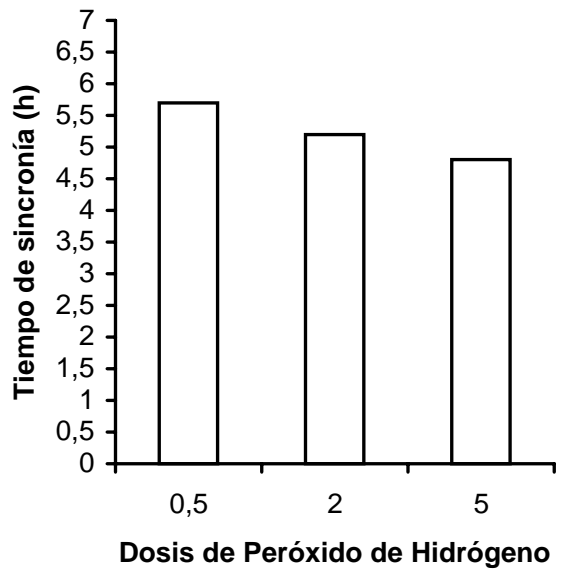

(\%)
Salinas Grandes de Hidalgo

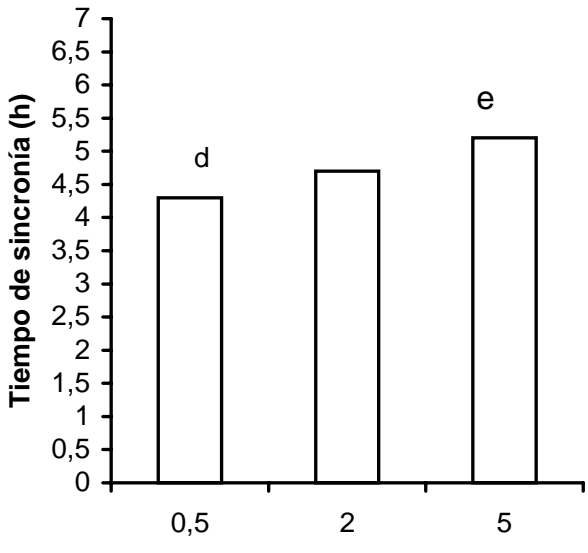

Dosis de Peróxido de Hidrógeno

(\%)

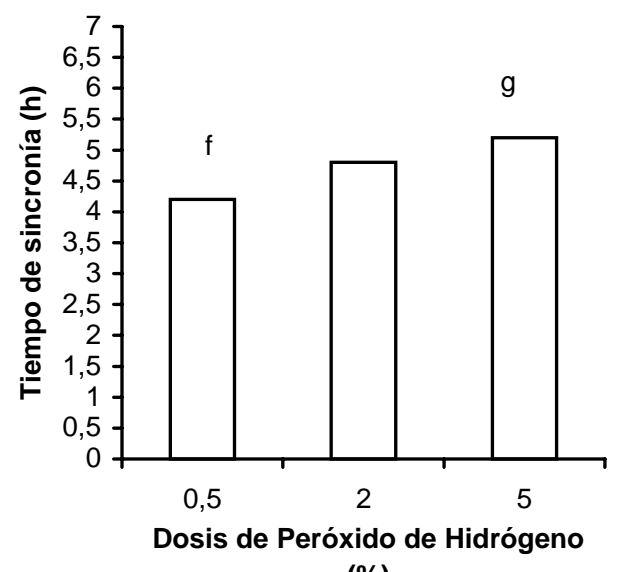

(\%)

Figura 3

Tiempo de sincronía (horas) de los quistes de Artemia persimilis provenientes de Salina del Inglés, Salinas Grandes de Hidalgo,

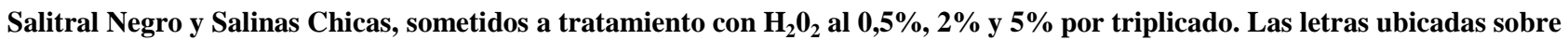
las barras señalan diferencias significativas $(p<0,05)$ entre tratamientos

Time of synchrony (hours) of Artemia persimilis cyst from Salina del Ingles, Salinas Grandes de Hidalgo, Salitral Negro and Salinas Chicas, treated with $0,5 \%, 2 \%$ and $5 \% \mathrm{H}_{2} \mathrm{O}_{2}$ in triplicate. Letters on the bars point out significant differences $(p<0,05)$ among treatments 
Salina Colorada Grande

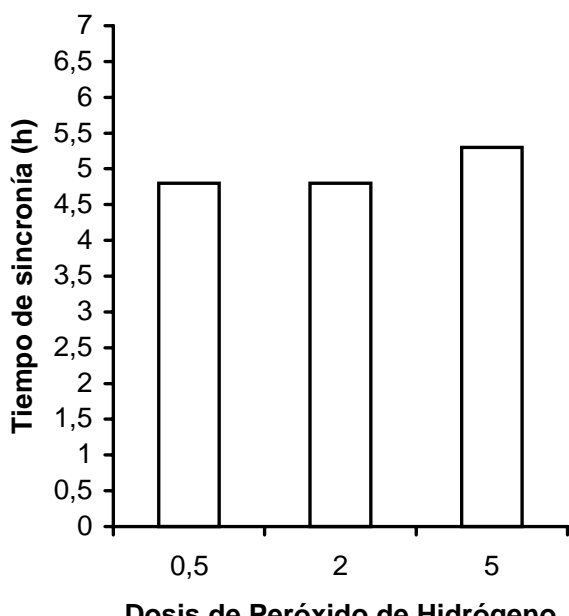

(\%)

Salina Colorada Chica

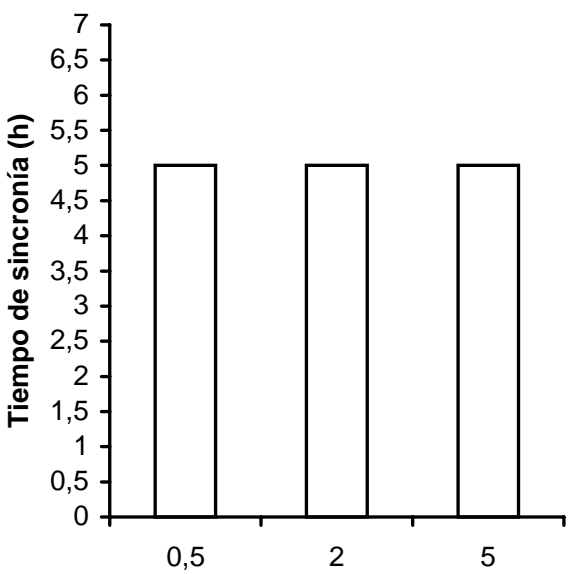

Dosis de Peróxido de Hidrógeno

(\%)
Salinas Grandes de Anzoátegui

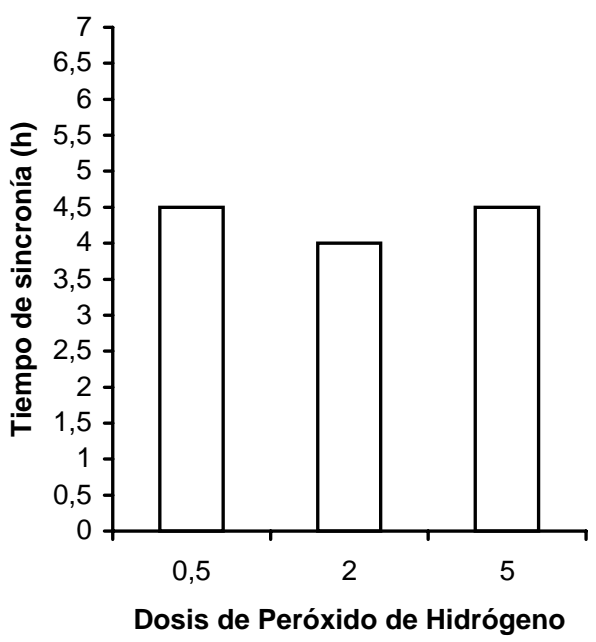

(\%)

Salinas Grandes de Córdoba

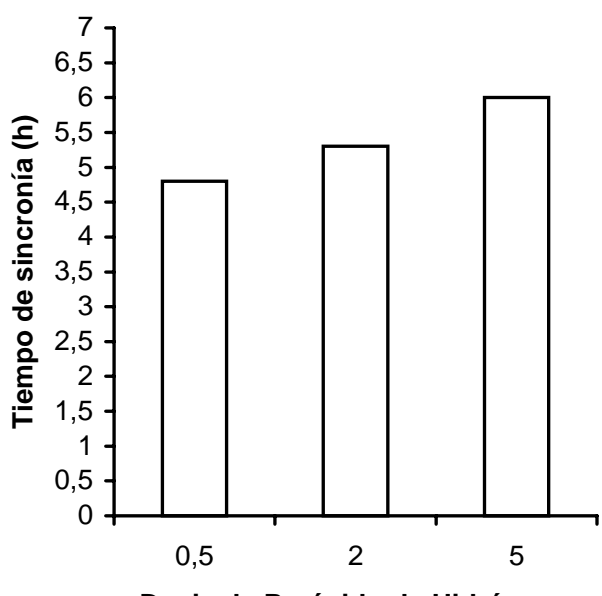

Dosis de Peróxido de Hidrógeno

(\%)

Figura 4

Tiempo de sincronía (horas) de los quistes de Artemia persimilis correspondientes a Colorada Grande, Salinas Grandes de Anzoátegui, Colorada Chica y Salinas Grandes de Córdoba,

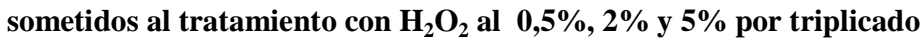

Time of synchrony (hours) of Artemia persimilis cysts corresponding to Colorada Grande, Salinas Grandes de Anzoategui, Colorada Chica and Salinas Grandes de Córdoba, treated with $0,5 \%, 2 \%$ and $5 \% \mathrm{H}_{2} \mathrm{O}_{2}$ in triplicate 
Tabla 3

Composición proximal en quistes de Artemia persimilis de tres salinas. Los datos corresponden a la media con su desvío estándar de tres réplicas

Proximal composition in cysts of Artemia persimilis from three salines. The data correspond to the average and standard deviation of three replicates

\begin{tabular}{ccccc}
\hline Salinas & Proteínas (\%) & Lípidos (\%) & Cenizas (\%) & Humedad (\%) \\
\hline Salinas Chicas & $56,2 \pm 1,70$ & $5,7 \pm 1,51$ & $6,7 \pm 0,55$ & $7,9 \pm 0,55$ \\
Salinas Grandes de Hidalgo & $55,3 \pm 1,72$ & $4,9 \pm 0,25$ & $5,5 \pm 0,55$ & $8,8 \pm 9,24$ \\
Colorada Chica & $56,7 \pm 1,73$ & $4,7 \pm 0,38$ & $4,6 \pm 0,11$ & $7,0 \pm 0,31$ \\
\hline
\end{tabular}

Tabla 4

Composición porcentual (\%) de ácidos grasos en quistes obtenidos de Salinas Chicas y Salinas Grandes de Córdoba

Percentage composition (\%) of fatty acids in cysts from Salinas Chicas and Salinas Grandes de Córdoba

\begin{tabular}{|c|c|c|c|}
\hline \multicolumn{2}{|c|}{ Salinas Chicas } & \multicolumn{2}{|c|}{ Salinas Grandes de Córdoba } \\
\hline Ácido graso & $(\%)$ & Ácido graso & $(\%)$ \\
\hline $14: 0$ & 0,7 & $14: 0$ & 0,90 \\
\hline $14: 1(n-5)$ & 2,6 & $14: 1$ & 0,79 \\
\hline 15:0 & 0,3 & $15: 0$ & 0,43 \\
\hline $15: 1(n-5)$ & 2,1 & $16: 0$ & 12,31 \\
\hline $16: 0$ & 11,4 & $16: 1$ & 6,94 \\
\hline $16: 1(n-7)$ & 6,8 & $17: 0$ & 1,15 \\
\hline 17:0 & 1,5 & $17: 1$ & 1,41 \\
\hline 18:0 & 4,6 & 18:0 & 4,72 \\
\hline $18: 1(n-7)$ & 13,0 & $18: 1^{*}$ & 26,55 \\
\hline $18: 2(n-6)-t$ & 9,2 & $18: 2(n-6)$ cis & 6,53 \\
\hline $18: 2(n-6)-c$ & 4,6 & $18: 3(n-6)$ cis & 0,58 \\
\hline $18: 3(n-3)$ & 23,1 & 18:3(n-3) & 19,62 \\
\hline $18: 3(n-6)$ & 0,8 & $18: 4(n-3)$ & 4,25 \\
\hline $18: 4(n-3)$ & 6,1 & 20:1 (n-9) & 0,22 \\
\hline 19:0 & 0,1 & $20: 4(n-3)$ & 0,92 \\
\hline 19:1 (n-9) & 0,7 & $20: 4(n-6)$ & 1,01 \\
\hline $20: 1(n-7)$ & 0,1 & $20: 5(n-3)$ & 0,61 \\
\hline 20:1 (n-9) & 0,3 & 22:6 & 0,21 \\
\hline $20.3(n-3)$ & 0,3 & & \\
\hline $20: 3(n-6)$ & 0,1 & & \\
\hline $20: 4(n-3)$ & 0,8 & & \\
\hline $20: 4(n-6)$ & 0,1 & & \\
\hline 20:5 (n-3) & 0,3 & & \\
\hline $22: 0$ & 0,2 & & \\
\hline $22: 5(n-3)$ & 0,1 & & \\
\hline $22: 5(n-6)$ & 0,2 & & \\
\hline 22:6 (n-3) & 0,2 & & \\
\hline 23:0 & 0,1 & & \\
\hline
\end{tabular}

* : Suma de isómeros 


\section{Discusión}

Según Lavens \& Sorgeloos (1996), entre los criterios más importantes que definen la calidad de los quistes de Artemia como alimento en acuicultura, están: la biometría de quistes y nauplios, porcentajes y tiempos de eclosión de los quistes y su valor nutricional.

Con relación a los diámetros promedios de los quistes, éstos variaron entre 184,7 $\pm 26,70 \mu \mathrm{m}$ (El Chancho) y 248,8 \pm 17,29 $\mu \mathrm{m}$ (Colorada Grande). Existe variación entre la talla de los quistes de las diferentes salinas analizadas. Alvarez \& Sánchez (1994) señalan que las condiciones ambientales son las responsables fundamentales de las diferencias observadas en las características biométricas tales como la talla de los quistes. Según Ortíz et al. (1991) existe variabilidad entre una cepa de Artemia y otra, pudiendo manifestarse en el tamaño de los quistes.

Con respecto al número de quistes por gramo de muestra seca y limpia, estos variaron entre 102.600 (Salinas Grandes de Anzoátegui) y 276.000 (Salinas Grandes de Hidalgo). Dichos valores son comparables a los quistes que se comercializan actualmente en el mercado internacional.

Según Lavens \& Sorgeloos (2000) las diferencias existentes en la calidad de la eclosión, pueden ser atribuidas principalmente a las condiciones ambientales, dado que los cambios medioambientales influencian en el estado de diapausa del quiste y esto finalmente, afecta la capacidad de eclosión de los mismos.

Los resultados obtenidos en esta investigación, mostraron bajos porcentajes de eclosión en quistes de Artemia persimilis procedentes de: El Chancho (42,0 $\pm 2,83 \%)$, Salinas Grandes de Annzoátegui $(44,0 \pm 6,93 \%)$ y Colorada Grande (45,3 $\pm 8,39 \%)$. Una explicación al fenómeno de que los quistes procedentes de estas salinas hayan presentado bajos porcentajes de eclosión en comparación con el resto, es que los mismos no sean de producción reciente, conteniendo una mezcla de edad diversa, acumulados en ocasiones diferentes y por lo tanto, habrían estado sometidos en distintos grados a la acción de la intemperie reduciendo de este modo la viabilidad. Según Rodríguez et al. (2002) se ha observado la acumulación de los quistes en las márgenes de distintas salinas a través del tiempo, indicando que estos quistes son un producto de varias generaciones.

En el resto de las salinas analizadas, los quistes presentaron porcentajes de eclosión elevados, que variaron entre 61,7 $\pm 7,64 \%$ (Salinas Grandes de Córdoba) y 82,0 $\pm 2,00 \%$ (Salinas Chicas, Provincia de Buenos Aires). Quynth et al. (1988) establecieron que los porcentajes de eclosión superiores al $70 \%$ son considerados como aceptables. De todos modos, en el mercado internacional se comercializan quistes de baja calidad, con el 50\% de eclosión o menos (Amat 1980).

De acuerdo con Castro (1993), el lapso entre la hidratación de los quistes y el $90 \%$ de eclosión, no debe exceder de las $29 \mathrm{~h}$ para que de esta forma, no disminuya el contenido energético de los nauplios y la tasa de eclosión sea aceptable.

Los resultados obtenidos de los tiempos de sincronía de los quistes de las diferentes salinas, fueron en general muy similares. Los tiempos de sincronía no superaron las 7,5 h, es decir, que eclosionaron de manera rápida y sincrónica, característica importante en la determinación de la calidad de los quistes.

Trabajos realizados por Van Stappen et al. (1998) indican que el tratamiento con peróxido de hidrógeno para lograr la desactivación de la diapausa, resulta efectivo en quistes de Artemia franciscana procedentes de la bahía de San Francisco (Estados Unidos de América) y de acuerdo con Clegg \& Jackson (1998) aún es desconocido el modo de acción del peróxido de hidrógeno en los quistes de Artemia. Según Lavens \& Sorgeloos (1996) es difícil predecir la sensibilidad de las cepas de Artemia al producto. Por lo tanto, son necesarios estudios para obtener información sobre la determinación de la dosis óptima y de esta manera, pueda lograrse un máximo efecto en la disminución de los tiempos de eclosión.

Se puede afirmar que los quistes de Artemia persimilis responden al tratamiento con peróxido de hidrógeno, mejorando los tiempos de sincronía, pero no de igual manera, dependiendo de la dosis aplicada. Cuando los tiempos de sincronía en los quistes son bajos, indicativo de una mejor calidad, parecería que se requieren dosis menores para mejorar los tiempos de eclosión. En cambio, con quistes de menor calidad, se deben aplicar dosis mucho mayores para lograr el efecto deseado. Según Drinkwater \& Clegg (1991), los métodos de desactivación de la diapausa son específicos para cada especie, cepa o incluso lote de quistes, es decir, que los quistes sometidos a tratamientos de desactivación de la diapausa, pueden responder de manera muy distinta o incluso, no presentar respuesta alguna.

Los quistes de Artemia persimilis mostraron, en general, valores nutricionales ligeramente menores pero 
aceptables y comparables a los quistes que se comercializan actualmente en relación al contenido de proteínas, humedad, cenizas y lípidos totales.

El contenido de ácidos grasos es un factor importante para la utilización de Artemia como alimento larval, especialmente en organismos marinos (Ishizaki et al. 2000). Algunos autores como (Fenucci et al. 1981, Coutteau \& Mourente 1997) indican que el ácido linolénico y el ácido linoleico son importantes para un buen desarrollo de las larvas de peces y crustáceos, bajo condiciones de cultivo. Según Van Stappeen (1996) los quistes de Artemia difieren en la composición de ácidos grasos. Todas las cepas de Artemia contienen ácido linolénico (18:3n-3) pero no todas contienen el ácido eicosapentanoico (20:5n-3) y ninguna contiene el ácido docosahexaenoico (22:6n-3) más que en cantidades trazas (Navarro et al. 1993). Watanabe et al. (1987) distingue dos grupos generales: Artemia tipo dulceacuícola y Artemia tipo marina. El primer grupo se caracteriza por presentar cantidades elevadas del ácido linolénico (18:3n-3) y niveles reducidos del ácido eicosapentanoico (20:5n-3). El segundo grupo contiene cantidades mínimas del ácido linolénico y niveles elevados del ácido eicosapentanoico. Navarro et al. (1993) determinaron los porcentajes de ácidos grasos en quistes de Artemia, correspondiente al tipo marino (quistes procedentes de Torrevieja, España) y al tipo dulceacuícola (quistes procedentes del Gran Lago Salado, Estados Unidos), según la clasificación anteriormente mencionada. Para los quistes del tipo dulceacuícola se obtuvieron: $0 \%$ (ácido eicosapentanoico) y 11,9\% (ácido linolénico). Para quistes correspondiente al tipo marino se obtuvieron: 6,5\% (ácido eicosapentanoico) y 2,7\% (ácido linolénico). Los resultados de la composición de ácidos grasos en los quistes de Artemia persimilis mostraron un contenido de 0,3\% (ácido eicosapentanoico, 20: 5n-3) en Salinas Chicas y de 0,61\% (ácido eicosapentanoico, 20: 5n-3) en Salinas Grandes de Córdoba. En ambas salinas analizadas, se detectaron cantidades importantes de ácido linolénico entre 19,6\% (Salinas Grandes de Córdoba) y 23,1\% (Salinas Chicas).

El conocimiento del tamaño naupliar resulta fundamental para su uso en acuicultura, debido a que muchas especies de larvas de organismos acuáticos, poseen bocas muy pequeñas y por lo tanto, es particularmente crítico el tamaño de la presa (Lavens \& Sorgeloos 1996). Los valores obtenidos, fueron en general menores en comparación a los nauplios de Artemia franciscana provenientes del Gran Lago Salado (Estados Unidos de América), cuya talla promedio es de 489,0 \pm 29,2 um (Castro et al. 1997). Según Vos et al. (1984) las diferencias en las tallas naupliares de las distintas cepas de Artemia, pueden deberse a diferencias en las condiciones ambientales. Amat (1985) expresa que los quistes sometidos a excesivos ciclos de activación y desactivación metabólica, pueden manifestar una disminución de la viabilidad de los quistes, fenomenología que puede concretarse en tamaños de nauplios progresivamente menores.

\section{Conclusiones}

Los resultados obtenidos en este trabajo confirman que los quistes de Artemia persimilis son de muy buena calidad y comparables a los quistes que se comercializan en el mercado internacional.

Los resultados de la composición en ácidos grasos indican que Artemia persimilis corresponde al tipo dulceacuícola y en caso de emplearse en larvicultura de especies marinas, los nauplios de esta especie deben someterse eventualmente a procesos de enriquecimiento con emulsiones lipídica.

\section{Agradecimientos}

Agradecemos la colaboración del Lic. Daniel Hernández, por su importante aporte en lo referente a temas estadísticos, a la cartógrafa Virginia Bernasconi por su valiosa colaboración en este trabajo y a la profesora María Elena Parodi por su importante aporte en la traducción al idioma inglés. Por último, un agradecimiento enorme a todos los que de alguna manera u otra, han hecho posible la concreción de este trabajo, en especial a la Dra. María Delia Viñas y a la Lic. Nora Harán.

\section{Literatura citada}

Alvarez Z \& R Sánchez. 1994. Variaciones en la calidad de la eclosión de la cepa de Artemia Las CumaraguasParaguana, Venezuela. Actas del VIII Congreso Latinoamericano de acuicultura de Santa Fe de Bogotá, Colombia, pp. 562.

Amat F. 1980. Antecedentes, estado actual y perspectivas del empleo de Artemia salina en acuicultura. Informe Técnico del Instituto de Investigaciones Pesqueras 75: 3-75.

Amat F. 1985. Biología de Artemia. Informe Técnico del Instituto de Investigaciones Pesqueras 127: 3-59.

Angelelli VI, B Schalamuk \& A Arrospide. 1976. Los yacimientos no metalíferos y rocas de aplicación de la región Patagonia-Comahue. Anales XVII. Ministerio de Economía. Secretaría de Estado de Minería. República Argentina, $147 \mathrm{pp}$. 
Angelelli VI, B Schalamuk \& R Fernández. 1980. Los yacimientos no metalíferos y rocas de aplicación de la región Centro-Cuyo. Anales XIX. Ministerio de Economía. Secretaría de Estado de Minería. República Argentina, 261 pp.

Barnes H. 1959. Apparatus and methods of Oceanography. George Allen and Unwin Ltd. New York, 339 pp.

Bossier P, W Xiaomei, F Catania, S Dooms, G Van Stappen, E Naessens \& P Sorgeloos. 2004. An RFLP database for authentication of commercial cyst samples of the brine shrimp Artemia spp. (International Study on Artemia LXX). Aquaculture 231: 93-112.

Castro BT. 1993. Biología y cultivo de Artemia franciscana en el Ex Lago de Texcoco de Ecatepec. Estado de México. Tesis doctoral. Universidad Nacional Autónoma de México, Facultad de Ciencias, 72 pp.

Castro BT, RC Gallardo, MJ Castro \& SA Malpica. 1997. Importancia del tamaño de los quistes descapsulados y de nauplios de Artemia franciscana (Texcoco) para la alimentación de organismos acuáticos. Revista de Investigaciones Marinas 18(2): 150-154.

Castro BT, MJ Castro, VRE Marin, GR Young, D Jenoure, MG Castro, SA Malpica \& AR De Lara. 2000. Calidad de quistes de Artemia franciscana (Kellogg, 1906) de la Laguna Pequeña de Yallahs. Jamaica. Ciencias Marinas 26(2): 201-214.

Clegg JS \& SA Jackson. 1998. The metabolic status of quiescent and diapause embryos of Artemia franciscana (Kellogg). Archiv für Hydrobiologie Special Issues Advances in Limnology 52: 425-439.

Cohen RG, F Amat, F Hontoria \& JC Navarro. 1999. Preliminary characterization of some Argentinean populations of the brine shrimp Artemia (Crustacea, Branchiopoda, Anostraca). Provinces of La Pampa and Buenos Aires. International Journal of Salt Lake Research 8: 329-340.

Coutteau P \& G Mourente. 1997. Lipid classes and their content of n-3 highly unsaturated fatty acids (HUFA) in Artemia franciscana after hatching, HUFA-enrichment and subsequent starvation. Marine Biology 130 (1): 81-91.

Dargám RM. 1995. Geochemistry of waters and brines from the Salinas Grandes basin, Córdoba, Argentina. International Journal of Salt Lake Research 3: 137-158.

De los Ríos P \& G Gajardo. 2002. Situación actual del conocimiento de poblaciones chilenas de Artemia spp. (Crustacea, Anostraca): ¿Es factible su uso en acuicultura? $\mathrm{X}$ Congreso Latinoamericano de Acuicultura, Santiago, Chile, p. 48.
Drinkwater LE \& JS Clegg. 1991. Experimental biology of cyst diapause: 93-117. En: Browne RA, P Sorgeloos \& CAN Trotman (eds) Artemia Biology. CRC Press, Boca Raton, Florida.

Fenucci JL, ZP Zein-Eldin \& AL Lawrence. 1981. The effects of fatty acid and shrimp meal composition of prepared diets on the growth of laboratory reared Penaeus stylirostris. Journal of the World Mariculture Society 12(1): 315-324.

Horwitz W. 1960. Official Methods of analysis of the Association of Official Agricultural Chemists. Association of Official Analytical Chemists, Washington, 325 pp.

IRAM. 1985. Método de determinación de la materia grasa por la técnica de extracción en un aparato tipo Soxhlet o Twiselmann. Instituto Argentino de Racionalización de Materiales, Norma IRAM 15040-1:7.

Ishizaki Y, K Uematsu \& T Takeuchi. 2000. Preliminary study of the effect of dietary docosahexaenoic acid on the volumetric growth of the brain in larval yellowtail. Fisheries Science 66: 611-613.

Lavens P \& P Sorgeloos. 1996. Manual on the production and use of live food for aquaculture. FAO, Rome, pp. 79251.

Lavens P \& P Sorgeloos. 2000. The history, present status and prospects of the availability of Artemia cysts for aquaculture. Aquaculture 181: 397-403.

Moreno VJ \& JE Aizpun. 1969. Manual de métodos de análisis de harina de pescado. Comisión Asesora Regional De Pesca Para El Atlántico Sudoccidental,12 pp.

Moreno VJ. 1977. Composición lipídica y biosíntesis de ácidos grasos de organismos del fitoplancton marino: Phaeodactylum triconutum (Bhlin) 1879 y Paracalanus parvus (Claus) 1863 respectivamente. Tesis doctoral, Facultad de Ciencias Exactas, Universidad Nacional de La Plata, 165 pp.

Navarro JC, RJ Henderson, LA McEvoy, MV Bell \& F Amat. 1999. Lipid conversions during enrichment of Artemia. Aquaculture 174: 155-166.

Navarro JC, F Amat \& JR Sargent. 1993. The lipids of the cysts of freshwater- and marine- type Artemia. Aquaculture 109: 327-336.

Ortíz F, M Sandoval \& G Araneda. 1991. Metodologías y recomendaciones técnicas para la cualificación y utilización de las cepas nativas de Artemia. Boletín Red Regional de Acuicultura de América Latina (Canadá) 5(3): 15-23.

Piccinelli M \& T Prosdocimi. 1968. Descrizione tassonomica delle due specie Artemia salina L. e Artemia persimilis n. sp. Istituto Lombardo. Classe Scienze 102: 113-118. 
Quynh VD, $P$ Lavens, $P$ Léger, W Tackaert \& $P$ Sorgeloos. 1988. Characterization of brine shrimp Artemia of Cam Ramh Bay in Central Vietnam. Hydrobiology 157: 209-217.

Rodríguez D, C Correa, J de la Rosa-Vélez, R Escobar, B Cordero, Z Alvarez \& R Sánchez. 2002. Variación alozimática en el crustáceo Artemia franciscana (Anostraca: Artemiidae) del Gran Lago Salado en varias condiciones experimentales. Revista de Biología Tropical 50(1): 87-95.

Schalamuk I, R Fernández \& R Etcheverry. 1983. Los yacimientos de minerales no metalíferos y rocas de aplicación de la región Noa. Anales XX. Ministerio de Economía. República Argentina, 208 pp.

Schalamuk I, M Blanco, D Marchioni, S Romero \& C Cábana. 1999. Salinas y Sulfateras de la Región Pampeana, Buenos Aires y La Pampa. Recursos Minerales de la República Argentina. Vol. II. Anales N ${ }^{\circ} 35$. Secretaría de Minería. República Argentina, pp. 19471962.

Sokal RR \& FJ Rohlf. 1979. Biometría. Blume Ediciones, $832 \mathrm{pp}$.
Van Stappen G. 1996. Introduction, biology and ecology of Artemia: 101-121 En: P Lavens \& P Sorgeloos (eds) Manual on the production and use of live food for aquaculture. FAO. Fisheries Technical Paper, $N^{\circ} 361$. Rome, Italy.

Van Stappen G, P Lavens \& P Sorgeloos. 1998. Effects of hydrogen peroxide treatment in Artemia cysts of different geographical origin. Archiv für Hydrobiologie Special Issues Advances in Limnology 52: 281-296.

Vos J, P Léger, P Vanhaecke \& P Sorgeloos. 1984. Quality evaluation of brine shrimp Artemia cysts produced in Asian saltponds. Hydrobiology 108: 17-23.

Watanabe T. 1987. The use of Artemia in fish and crustacean farming in Japan: 373-393. En: P Sorgeloos, DA Bengtson, W Decleir \& E Jaspers (eds). II International Symposium on the brine shrimp Artemia. Vol. III, Universa Press, Wetteren, Bélgica.

Zar HJ. 1984. Biostatistical Analysis. Second Edition. Prentice-Hall Inc, Englewood Cliffs, New Jersey, 718 pp. 